\title{
Mar adentro
}

\section{Erhard Taverna}

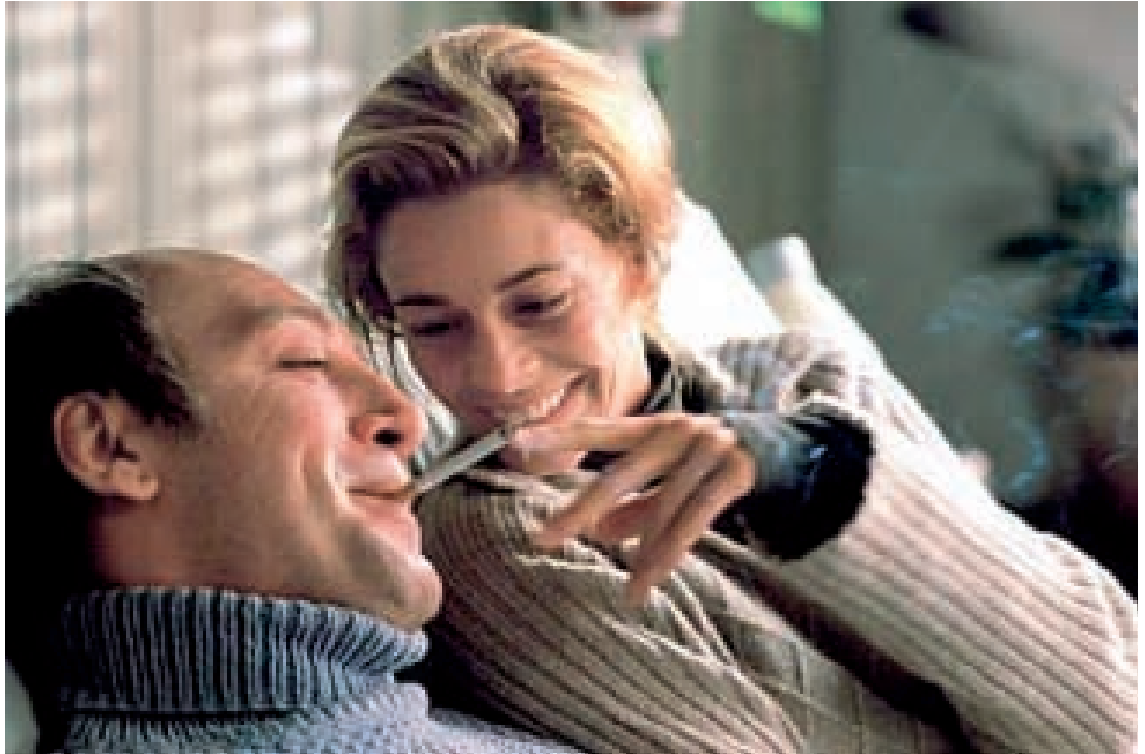

Der politisierte Tod der amerikanischen Komapatientin Terri Schiavo oder das inszenierte Sterben des Papstes wecken verschiedene Emotionen. Für die einen sind es Vorbilder und Lehrstücke, für andere nur Stoff für Voyeure, ein morbides Spektakel. Vielleicht nimmt sich die Fiktion der Realität an und schafft daraus einen Kinofilm, wie den des Regisseurs Alejandro Aménabar, der aus der wahren Geschichte des Spaniers Ramón Sampedro entstand, eines Paraplegikers, der gegen Staat und Kirche seinen selbstgewählten Tod durchsetzte. Der für zwei Oscars nominierte Film «Mar adentro» schafft es, diesen jahrzehntelangen Kampf um Sterbehilfe als schnörkellose, berührende, tiefsinnige und lebensfrohe Geschichte zu erzählen. Als Matrose fuhr Sampedro um die Welt, bis er sich bei einem Badeunfall das Genick brach. «Wenn man abhängig ist, lernt man zu lachen, statt zu weinen», erklärt er seiner Anwältin. Er war ein todernster Charmeur, ein verzweifelter Humorist, ein nüchterner Träumer, von vielen bis zur letzten Minute geliebt und umworben. Bis zum Ende, wo er das aufgelöste Zyankali durch einen Trinkhalm vor laufender Kamera einnimmt. Hervorragende Schauspieler und ein kluges Drehbuch haben die meisten Kritiker begeistert, und dass die Kirche in Spanien den Film scharf verurteilte, machte ihn dort zusätzlich zum Publikumsrenner.
Die moderne Medizin und die Beihilfe zum Suizid ist auch bei uns immer wieder ein Thema. Die Schweiz ist in dieser Beziehung toleranter als die umliegenden Länder. Wenn beim Helfer keine eigennützigen Motive vorliegen, ist die Suizidhilfe gemäss eidgenössischem Strafrecht seit 1948 nicht strafbar. Diese gesetzliche Formulierung wird seit einigen Jahren auch im Zusammenhang mit dem hochmedikalisierten Sterben verwendet, wobei auch die neuen medizinischethischen Richtlinien der SAMW es offenlassen, ob ärztliche Personen in Ausnahmefällen Sterbehilfe leisten dürfen. Eine Schwierigkeit besteht darin, dass sich die Probleme in unterschiedlichen Fachbereichen wie Neonatologie, Onkologie, Intensivmedizin, Geriatrie oder Allgemeinmedizin massgeblich voneinander unterscheiden.

Jährlich setzen in der Schweiz rund 1500 Menschen ihrem Leben ein Ende. Ausserhalb der Spitäler sind es etwa 300, die auf ihrem letzten Weg unterstützt werden, wobei in den meisten dieser Fälle fünf Sterbe- und Freitodhilfeorganisationen beteiligt sind: Exit (deutschsprachige Schweiz), ADMD (Suisse romande), Ex-International, Suizidhilfe und Dignitas. Da vor allem letztere einen «Suizidtourismus» ausgelöst hat, werden zurzeit im Kanton Zürich und bald auch in anderen Kantonen sowie auf Bundesebene Suizidbeihilfegesetze erarbeitet. Grundgedanke ist bis heute das liberale Recht auf die Beendigung des eigenen Lebens, wobei idealtypisch an einen rationalen Suizid, wie im Film, gedacht wird. Eine kompetente Person entscheidet sich in Kenntnis aller relevanten Faktoren für den Tod, und wie beim Recht auf Glaubens- oder Redefreiheit oder Eigentum würde es die Menschen in ihrer Würde verletzen, wenn ihnen die rationale Suizidhandlung verwehrt wäre. Keine Ethik kann im konkreten Einzelfall den betroffenen Patienten, Angehörigen, Pflegenden oder Ärzten die Entscheidung abnehmen.

Für den Gläubigen ist der selbstgewählte Tod ein Verstoss, eine Sünde. Ein tetraplegischer Priester versucht im Film, Ramon Sampedro von seinem Plan abzubringen. Für ihn ist das Leben ein Geschenk Gottes, das der Beschenkte nicht vernichten darf. Aus theologischer Sicht ist diese Verpflichtung zwingend, denn nur Gott allein entscheidet über den Zeitpunkt des Ablebens. 
Ramon hat die besseren Argumente, zudem verunglimpft der Priester die pflegenden Angehörigen. Als Nichtgläubiger weist der Verunfallte den Totalanspruch des Geistlichen zurück. Für ihn gibt es kein Leben nach dem Tode, so wenig wie vor der Geburt.

Der moderne Staat steht vor einem Dilemma. Während eines Prozesses, ausgelöst durch die mütterliche Suizidhilfe an ihrem vollständig gelähmten Sohn, der um die Erlaubnis bat, seinem Leben ein Ende zu setzen, formulierte der französische Premierminister Raffarin: «On ne peut pas gouverner ou légiférer sur des situations spécifiques. La vie n'appartient pas aux politiques.» Doch die Gesellschaft hat die Autonomie des einzelnen Menschen zu beachten und gleichzeitig das Leben zu schützen, im Wissen, dass die Welt der Glücklichen eine andere ist als die der Unglücklichen. Nur allzuleicht kann sich das Recht auf Suizid zu einer Pflicht verkehren. Das pragmatische Vorgehen der Schweiz ist vernünftig; man kann sehr wohl das eine tun und das andere nicht lassen.

\title{
Die Welle (Teil 2)
}

\section{Kurzgeschichte}

\author{
A. J. Koemeda
}

Korrespondenz:

Dr. med. Ada Jens Koemeda Holbeinstrasse 20 CH-8008 Zürich
Während einiger Jahre fuhr Astrid nicht mehr nach Ungarn. Ilonka, jetzt Biologiestudentin, zog 2004 aus, zunächst in eine Wohngemeinschaft, dann in ihre eigene kleine Wohnung. Am Anfang lebte sie darin alleine, später mit ihrem Freund, einem Sohn polnischer Emigranten; miteinander sprachen sie Baseldeutsch. Ihre Mutter besuchte sie natürlich, eher unregelmässig; einmal bis zweimal in der Woche telefonierten sie miteinander.

Astrid hatte nun mehr Zeit für sich; neben der Tätigkeit als Lehrerin und Therapeutin beteiligte sie sich an einem psychosozialen Forschungsprojekt. Als Wissenschaftlerin - sie dachte dieses Wort immer in Anführungsstrichen und schmunzelnd - war sie nur mässig erfolgreich, die Arbeit bereitete ihr aber viel Freude. Ab und zu wurde sie als Referentin zu Kongressen eingeladen, auch ins Ausland. Darüber freute sie sich besonders. Englisch konnte sie sehr gut, obwohl sie in Amerika nur einen Monat lang gelebt hatte, Französisch inzwischen auch, Italienisch fast gar nicht.

Kriege kamen, Terroristen verunsicherten zunehmend die nichtislamische Welt, die mächtigen Twins in New York stürzten wie schlechtgeplante Plattenbauten in einem Erdbebengebiet ein. Im Irak waren die Amerikaner wieder zugange, ihre Präzisionsmaschinen führten akkurate chirurgische Schnitte an wichtigen militärischen Einrichtungen des Feindes durch, die leider nicht immer akkurat genug waren: Den Tod von Tausenden Zivilisten - im Rahmen lästiger Kollateralschäden - nahm man aber in Kauf.

Den verwahrlost, abgebaut und betäubt wirkenden Ex-Peiniger des Landes hatte man den kurdischen Entführern für teures Geld abgekauft; bald danach wurde er, liebevoll und ausdrucksstark inszeniert, der Weltöffentlichkeit stolz gezeigt: Man zog den leicht verwirrten Alten aus einem Erdloch und die Amerikaner, nicht ahnend, was später noch alles kommen würde, klopften sich zufrieden auf die Schultern.

Ein halbes Jahr später entging Astrid nur knapp dem Tod. Sie wollte, zum ersten Mal nach vielen Jahren, Südfrankreich untreu werden und nach Malaysien fliegen. Die gewünschte Anzahlung wurde geleistet, der Koffer vom Schrank geholt und entstaubt, ein paar einschlägige Reisebücher besorgt und sorgfaltig studiert.

Und: Dann kam die Einladung!

Sie sollte doch nach Budapest gehen, einen erkrankten Kollegen vertreten, das heisst, sein Manuskript ein wenig straffen und dort selber vortragen. Und sie könne, wenn sie Lust dazu hätte, auch ihre eigene Arbeit kurz vorstellen («bitte, bitte, sagen Sie ja», liess sich der Chef hören, «wir finden doch in so kurzer Zeit keinen Ersatz!»).

Also: Nach Budapest. Oder doch nach Phuket, Badeferien?

Die Entscheidung fiel ihr nicht schwer, sie telefonierte sofort: Jaja, ich komme gerne, ich will niemanden im Stich lassen; ich werde in Budapest auf alle Fälle dabei sein! 
Man bedankte sich herzlich bei ihr und sprach von grosser Erleichterung. Sogar eine Schachtel Pralinen bekam sie noch am gleichen Abend!

Sie kürzte ihren Vortrag, las viel Fachliches und bereitete sich sorgfaltig auf eine eventuelle Diskussion mit dem Publikum vor.

Winteranfang. Der erste Schneesturm. Astrid hatte diesmal erwogen, mit dem Auto zu fahren; angesichts der Wetterlage entschloss sie sich aber, die Reisegefahren zu mindern und bestieg wieder den Zug. Im Hotel, das sie inzwischen «mein Hotel» nannte, bekam sie ohne Schwierigkeiten ein Zimmer; nicht das gleiche allerdings wie bei ihren letzten Reisen, sondern ein Stockwerk höher - für sie kein Problem!

Dass sie wieder zu telefonieren versuchen würde, daran zweifelte sie keine Sekunde; unklar war nur, ob sie es vor oder nach ihrem Kongressauftritt tun sollte. Da sie eine grössere Aufregung nach einem eventuellen Gespräch mit Mirko nicht ausschliessen konnte - und weil sie auch durch ein verpatztes Referat den erkrankten Kollegen nicht enttäuschen wollte, entschloss sie sich für einen weiteren Anrufversuch erst am letzten Kongresstag.

Abreise. Astrid packte langsam und sehr sorgfältig ihren Koffer - für sie ein wichtiges $\mathrm{Ab}$ schiedsritual. Dann lüftete sie gründlich das Hotelzimmer, stärkte sich mit einem Gin Tonic aus der eher bescheiden bestückten Minibar und legte sich aufs Bett.

Sie nahm das hellgraue Telefon in die Hand und wählte. Kein Besetztton, die Linie war frei, aber niemand nahm ab - wie vor zwei, drei Jahren. Astrid war weder überrascht noch besonders traurig, irgendwie hatte sie damit gerechnet. Später rief sie die Tochter an, sie wollte ihr nur hallo sagen und nach den letzten Neuigkeiten fragen; dennoch wurde aus dem Telefonat ein fast halbstündiges Gespräch.

Sie legte auf. Lange, zu lange haben wir geredet, dachte sie, zu teuer. Und trotzdem - notwendig. Sie war zufrieden.

Astrid schaute auf die Uhr. Zeit hatte sie noch genug. Was also jetzt? Hinaus, noch eine Abschiedsrunde in der Stadt drehen, ein wenig Bewegung vor der langen Zugreise? Eine Minishoppingtour, ein paar Geschenke besorgen? Sie war unentschlossen. Vielleicht doch nur in die Hotelbar im Erdgeschoss. Ja, lieber! Vorher allerdings noch einen, den letzten Versuch bei Mirko. 0131958 ... plötzlich wusste sie nicht weiter, sie schüttelte den Kopf. Schnell holte sie ihre Agenda aus der Tasche und begann, die Nummer zu suchen. Es ging nicht so rasch, wie sie dachte, die schon eingeleitete Verbindung wurde unterbrochen; kurze, hohe Töne piepsten ihr ins $\mathrm{Ohr}$ - sie musste auflegen und mit dem Wählen von vorne anfangen. Nur langsam, ganz langsam, sagte sie sich, keine Aufregung.

- Hallo ... Eine Kinderstimme!

Dass Mirko abnimmt, ja, damit hatte Astrid immer noch ein wenig gerechnet, oder eine Frau; nicht aber ein Kind.

- Ja, ich wollte ... eigentlich wollte ich mit Herrn Nemeth sprechen.

- Papa ist nicht da!

- Ach so!

- Soll ich Mama rufen?

- Nein, nein. Danke!

- Papa kommt aber bald.

- Ja, danke.

Als Astrid das Telefon fast schon auf der Gabel hatte, hörte sie aus der grauen Muschel:

- Wie heisst...

Zu spät! Sie konnte nicht mehr bremsen. Aufgelegt!

Was nun? Sie überlegte ein paar Sekunden, dann rief sie noch einmal an. Das Mädchen nahm sofort ab.

- Du hast zum Schluss noch gefragt, wie ich heisse. Astrid, Astrid heisse ich.

- Ach so ... Astrid, wiederholte das Kind. Tschüss!

Astrid legte auf und blieb noch eine Weile auf dem Bett liegen; die Tränen konnte sie aber nicht mehr zurückhalten. Einige Minuten später stand sie schnell auf, griff nach ihrem Koffer und ihrer Handtasche und verliess das Zimmer. Einen kleinen Geldbetrag - das hatte sie früher immer gemacht - vergass sie diesmal auf den Nachttisch zu legen.

Anderthalb Stunden später sass sie schon im Zug; Nichtraucherwaggon. Noch auf ungarischem Boden baute der Schaffner die Lehn- und Sitzflächen zu Schlafliegen um. Von den sechs schmalen Betten waren nur fünf besetzt; drei Frauen und zwei Männer. Astrid war froh darüber, ein umgekehrtes Verhältnis hätte sie irritiert. Als Sprache des Abteils kristallisierte sich bald Deutsch heraus; neben ihr verstand nur der jüngere der beiden Männer Ungarisch.

Oft hatte Astrid von den Budapest-München-Zugreisenden Horrorgeschichten gehört; keine Morde, kein Blutvergiessen, nur Raubstorys - gutorganisierte Profileistungen: bis auf Schlaf- oder Trainingsanzug, den man nur die Nacht gerade anhatte, alles weg!

Sie nahm sich deshalb vor, die ganze Nacht wach zu bleiben und liess sich Kaffee bringen. Dann schlug sie ihr Buch auf: Höchstens eine 
Stunde, dachte sie, mehr liegt nicht drin. Die Augen fielen ihr immer wieder $\mathrm{zu}$, drei-, viermal las sie dieselbe Zeile. Nichts zu machen, hoffnungslos! Sie gab auf.

Als Kind war sie zwar mit den Eltern oft zum Balatonsee gereist, war allerdings nie eine Wasserratte. Im Traum wusste sie - nein, sie ahnte es nur -, dass sie träumte, und sie wunderte sich sehr, wovon: Eine riesige Welle kam auf sie zu, still, fast geräuschlos und hob sie in die Höhe, vorsichtig und weich. Im ersten Augenblick hatte sie Angst, als sie aber merkte, wie ruhig und behutsam sie die warme Welle trug, verlor sie ihre Bedenken. Und dennoch, geniessen konnte sie die ungewöhnliche Fahrt kaum; die Angst verliess sie zwar mit der Zeit, statt dessen überfiel sie aber eine merkwürdige, vor allem in der Brust und im Herzen steckende Trauer.

Astrid verstand es nicht, der Wellenritt war doch wunderbar: Sie sah unter sich grosse Städte, Wälder, Flüsse, der schmale Strich in der Landschaft war wahrscheinlich eine Autobahn. Sie schaute fasziniert auf den Boden, auf die tief unter ihr liegende Welt, sie wollte noch mehr von ihr erfahren, sie blickte nach links und nach rechts und weit nach vorne ... ja, sie bekam das Gefühl, die Erhebungen vor ihr, am vorderen Rand der Welle, seien die ersten voralpinen Bergketten.

Sie wollte sich noch umdrehen und sehen, was von dem flachen Land zwischen dem Neuenburgersee und Budapest zu erkennen wäre; sie vermochte es aber nicht zu tun, denn der Hals schmerzte und der Nacken wurde hart und steif. Warum kann ich mich nicht bewegen?, fragte sie sich, was ist los mit mir? Keine Antworten fielen ihr ein, nur Ärger stieg in ihr auf und dann plötzlich Angst: Sie konnte sie nicht abwälzen, sich von ihr befreien, sie konnte nicht einmal Luft holen ... jetzt naht das Ende, sagte sie sich; ich bin im Zug und dennoch im Wasser ... am Ertrinken. Sie wollte husten, fand allerdings nicht genug Kraft dazu.

So kann es nicht weitergehen, dachte sie; die Welle läuft doch nicht um den ganzen Erdball, vorher würde sie sich verbrauchen, vorher verliert sie ihren Schwung, ihre Fähigkeit, mich zu tragen ... wo werde ich aber landen, was werde ich tun; und wie kann ich dann noch weiterleben?

Astrid erwachte. Der Zug ging in eine Kurve und drückte sie an die glatte, hellbeige Kunststoffwand. Sie griff unter das Kissen, die Handtasche mit Geld und Dokumenten lag immer noch an ihrer Stelle. Sie klemmte sie unter den Arm und ging zur Toilette; der Gang war leer, das WC frei und sauber. Als sie wieder unter die Decke kroch, schaute sie schnell auf die Uhr bald halb drei. Der Zug zählte mit der Geduld und Genauigkeit eines Roboters die Nahtstellen an den Schienen und die Elektromaste auf der sanften Bahnböschung.

Astrid erinnerte sich an die Welle und war kurz glücklich, gleich aber auch enttäuscht darüber, dass sie nur im Traum auf dieser Riesenwelle reiten durfte. Und dennoch war sie froh um den Traum, denn er zeigte und bestätigte ihr, was sie schon seit einigen Stunden ahnte: Den Weg zurück will sie nicht mehr weitersuchen ... den gibt es für sie nicht mehr.

Wieder einschlafen konnte sie jetzt nicht. Sie versuchte es zwar ein paar Minuten lang, dann gab sie auf und knipste die kleine Leselampe an der Wand an. Eigenartigerweise dachte sie keine Sekunde daran, ihr Buch aufzuschlagen. Sie schaute aus dem Fenster in die Nacht, weinte wieder eine Weile und schloss die Augen. Sie spürte Wärme im ganzen Körper, ohne dass sie sich zugedeckt hatte, Wärme und Ruhe. Tak, tak, tak ... tak, tak, tak. Der Rhythmus des nächtlichen Zuges erinnerte sie an ein Lied, an eine alte, vertraute Melodie, sie konnte aber nicht sagen, ob das ein österreichisches, schweizerisches oder ungarisches Lied war.

Drei vorbei. Kein Schlaf mehr; endgültig!

Sie sah wieder aus dem Fenster und versuchte, der Dunkelheit etwas zu entreissen oder zumindest zu erfahren, ob der Zug in der Ebene ratterte oder an bewaldeten Berghängen vorbeihuschte.

Eine halbe Stunde später lag sie immer noch wach. Plötzlich kamen ihr verschiedene Möglichkeiten des Einschlafens in den Sinn, aber am längsten beschäftigte sie die radikalste Variante, einschlafen ohne zu erwachen; sie fand allerdings mehrere Gründe - Ilonka und ihre Arbeit standen an erster Stelle -, um sie schnell wieder $\mathrm{zu}$ verwerfen beziehungsweise um sie nur als die letzte, die «Reservelösung», zu betrachten.

Nein, ganz andere, viel optimistischere Varianten fielen ihr in dem Moment nicht ein, nicht so schnell. Sie ahnte zwar, dass es sie gab, und es ärgerte sie, dass ihr in diesem Augenblick keine einzige in den Sinn kam.

Alles ist nun offen, nichts ist geregelt, dachte sie kurz vor dem Einschlafen und fand es schrecklich. Schrecklich, aber auch schön. 